\title{
Solitary Waves of Ultra Intense Laser Pulses in a Fully Relativistic Plasma Channel
}

\author{
Ebrahim Heidari and Morteza Aslaninejad
}

\begin{abstract}
We present a theoretical study of nonlinear interactions between a short, intense laser-beam and a fully relativistic plasma channel containing hot electrons and positrons. We have obtained the governing equation for the evolution of the spot size with relativistic corrections. Also, the acceptable solutions of relativistic solitary waves have been predicted.
\end{abstract}

Index Terms-Laser-plasma interactions, Plasma channel, Solitary waves.

\section{INTRODUCTION}

The development of ultra intense short laser pulses allows exploration of fundamentally new parameter regimes for nonlinear laser plasma interaction [1]-[3]. Now we have laser beams with intensities up to $10^{21} \mathrm{~W} / \mathrm{cm}^{2}$ in laboratories. At such a high intensities electrons quiver velocity becomes relativistic. In such a situation, consideration of the relativistic electron mass variation [4]-[8] as well as the relativistic ponderomotive force [9], [10] is very essential in the study of nonlinear laser plasma interactions.

When an ultra intense laser pulse propagates in plasma, several nonlinear phenomena are excited, as, for example, laser frequency variation, high-order harmonic generation, the appearance of coherent nonlinear structures (plasma channels, relativistic solitons, vortices), the generation of ultra intense quasistatic electric and magnetic fields, and electron and ion acceleration to relativistic energies.

The paper is organized as follows. In section 2, we present the relevant equations to formulate the proposed problem with appropriate assumptions and the relativistic versions of the equations describing the evolution of the spot size is obtained. In section3, we discuss the solution to the governing equation. Section 4, summarizes the findings of this study. It is necessary to mention that, in the low velocity limit, all the expressions found in this study reduce exactly to their weak relativistic expressions [11].

Manuscript received April 20, 2012; revised June 4, 2012.This work was supported by the office of vice chancellor for research of Islamic Azad University, Bushehr Branch.

E. Heidari is with Department of Sciences, Bushehr Branch, Islamic Azad University, Bushehr, Iran (e-mail: ehphys75@ yahoo.com).

M. Aslaninejad is with Plasma Physics Research Centre, Science and Research Branch, Islanic Azad University, Tehran, Iran (e-mail: morteza@theory.ipm.ac.ir).

\section{BASIC EQUATIONS}

We first present the relevant equations describing the action of intense laser light on the plasma consisting hot electrons and positrons. We assume a circularly polarized laser pulse propagating along the $z$-direction in a hot and under dense plasma channel which has a parabolic density profile of the form $n(r)=n_{0}\left(1+r^{2} / r_{c h}^{2}\right)$, where $n_{0}$ and $r_{c h}$ are the initial axial electron density and the effective channel radius, respectively. By the ansatz,

$$
\mathbf{a}(r, z, t)=\frac{1}{2} a(r, z, t)(\hat{x}+\mathrm{i} \hat{y}) \exp \left[\mathrm{i}\left(k_{0} z-\omega_{0} t\right)\right]
$$

where $a(r, z, t)$ is the complex amplitude normalized by $m_{0} c^{2} / e, m_{0}$ and $e$ are the rest mass and the charge of electron, $c$ is the light velocity in vacuum and $k_{0}$ and $\omega_{0}$ are the laser centre wave number and the frequency, respectively, we can obtain the wave equation as

$$
\begin{aligned}
& \left(\nabla_{\perp}^{2}+2 \mathrm{i} k_{0} \frac{\partial}{\partial z}-k_{p}^{2} \eta \frac{r^{2}}{r_{c h}^{2}}+k_{p}^{2} \frac{|a|^{2}}{2}\right. \\
& \left.-\eta^{2} k_{p}^{2} \nabla_{\perp}^{2} \frac{|a|^{2}}{2}\right) a(r, z)=0
\end{aligned}
$$

where $k_{p}=\omega_{p} / c$ is the plasma wave number and $\eta$ is defined as $\eta=\gamma_{V} \frac{1-\beta_{0} v}{\gamma_{0}\left(\beta_{0}-v\right)^{2}}$. Also, $\beta=\frac{u}{c}, v=\frac{V}{c}$ and $\gamma_{V}=1 /\left(1-V^{2} / c^{2}\right)^{1 / 2}$ is the 'effective relativistic factor' associated with the velocity of the wave and should not be confused with the relativistic factor, $\gamma=1 /\left(1-u^{2} / c^{2}\right)^{1 / 2}$, related to the fluid velocity of the plasma. The subscript 0 represents quantities at infinity. In deriving equation (2), we have used the Coulomb gauge $\nabla \cdot \mathbf{a}=0$ and the dispersion relation $\omega_{0}^{2}=\omega_{p}^{2}+k_{0}^{2} c^{2}$.

Also, the paraxial approximation and slowly varying envelope approximation have been used. We note that in the limit $\beta_{0} \rightarrow 0$ (i.e. $\gamma_{0} \rightarrow 1$ ), equation (2) approaches its weak relativistic format in [11]. Using equation (2) and assuming that this equation has a solution as 


$$
a(r, z)=a_{r}(z) e^{-r^{2} / r_{s}^{2}(z)} e^{\mathrm{i}\left[b(z) r^{2}+\phi(z)\right]}
$$

where $a_{r}(z), r_{s}(z), b(z)$ and $\phi(z)$ are the real amplitude, spot size, spatial chirp parameter and phase shift of the laser pulse respectively, after straightforward, but tedious derivations, equation (2) can be written as

$$
\frac{1}{2}\left(\frac{\partial r_{s}}{\partial z}\right)^{2}+\psi\left(r_{s}\right)=0
$$

where

$$
\begin{aligned}
& \psi\left(r_{s}\right)=\frac{1}{2}(1-p)\left[r_{s}\left(\beta_{0}-v\right)\right]^{-2}\left(1-\beta_{0} v\right)+\frac{1}{2} v^{2} N_{c} r_{s}^{2} \\
& \times\left[\left(\beta_{0}-v\right)\right]^{-2}-\frac{1}{8} \gamma_{0}\left(1-\beta_{0} v\right) a_{0}^{2} r_{s}^{-4}-\psi_{0} \gamma_{0}
\end{aligned}
$$

Here, $\psi_{0}=\frac{1}{2}(1-p) \frac{1-\beta_{0} v}{\left(\beta_{0}-v\right)^{2}}+\frac{1}{2} v^{2} N_{c}-\frac{\gamma_{0} a_{0}^{2}}{8}$, $p=k_{p}^{2} a_{0}^{2} r_{0}^{2} / 16$ is the normalized laser power by the critical power $P_{c}=16 \pi$ for relativistic self- focusing in plasma and $N_{c}=\gamma_{0}^{2} k_{p}^{2} r_{0}^{4} / 4 r_{c h}^{2}$ is a parameter related to the effect of reformed channel focusing. In equations (4) and (5) the dimensionless variables $z / Z_{R} \rightarrow z$ and $r_{s} / r_{0} \rightarrow r_{s}$ where $Z_{R}=k_{0} r_{0}^{2} / 2$ is the Rayleigh length, are used. We also note that, a collimated incident laser pulse, i.e., $b_{0}=\left(\frac{\partial r_{s}}{\partial z}\right)_{z=0}=0$ and the initial condition $r_{s}=1$ at $z=0$ in integrating equation (4) is considered. Equations (4) and (5) are the relativistic versions of the equations describing the evolution of the spot size. It is interesting to note that for $\beta_{0}=0$ equations (4)-(5) obviously reduce to the expressions for the weak relativistic limits [11].

\section{ACCEPTABLE SOLUTIONS OF RELATIVISTIC SOLITARY WAVES}

Now we are going to turn our attention to invstigate the existence of solitary wave solutions of equation (4). We follow the same technique used in [11]. The roots of $\psi\left(r_{s}\right)=0$ can be easily found using the solutions of a cubic equation. The roots, in terms of the coefficient can be expressed as

$$
\begin{gathered}
r_{s 1}=1 \\
r_{s 2}=\left[\left(N_{p}+\sqrt{N_{p}^{2}-16 N_{c} a_{0}^{2}}\right) / 8 N_{c}\right]^{1 / 2} \\
r_{s 3}=\left[\left(N_{p}-\sqrt{N_{p}^{2}-16 N_{c} a_{0}^{2}}\right) / 8 N_{c}\right]^{1 / 2}
\end{gathered}
$$

where

$$
N_{p}=4(1-p) \eta-\gamma_{0}^{2} a_{0}^{2}
$$

Real and positive roots are acceptable. Different cases can be considered as,

(a) if $p>1-\gamma_{0} a_{0}^{2} \sqrt{N_{c}}-\gamma_{0}^{2} a_{0}^{2} / 4$, the equation has one real (i.e. $r_{s 1}=1$ ) and two non real complex conjugate roots.

(b) if $p<1-\gamma_{0}^{2}\left(N_{c}-a_{0}^{2} / 2\right), \psi\left(r_{s}\right)=0$ has three distinct real roots $r_{s 3}<r_{s 1}=1<r_{s 2}$.

(c)

$1-\gamma_{0}^{2}\left(N_{c}-a_{0}^{2} / 2\right)<p<1-\gamma_{0} a_{0}^{2} \sqrt{N_{c}}-\gamma_{0}^{2} a_{0}^{2} / 4$, three cases can be considered.

(c1) if $N_{c}=N_{c}^{*}$, where the critical channel parameter $N_{c}^{*}=\gamma_{0}^{2} a_{0}^{2} / 4 \quad, \quad \psi\left(r_{s}\right)=0$ has triple root, i.e., $r_{s 1}=r_{s 2}=r_{s 3}=1$.

(c2) if $N_{c}>N_{c}^{*}$, three kinds of cases are discussed as follows:

(c2.1) if $p=1-\gamma_{0} a_{0}^{2} \sqrt{N_{c}}-\gamma_{0}^{2} a_{0}^{2} / 4, \psi\left(r_{s}\right)=0$ has three real roots: $r_{s 1}=1$ and twofold root $r_{s 2}=r_{s 3}=\sqrt{a_{0} / 2 \sqrt{N_{c}}}<1$.

(c2.2) if $1-\gamma_{0}^{2}\left(N_{c}-a_{0}^{2} / 2\right)<p<1-\gamma_{0} a_{0}^{2} \sqrt{N_{c}}-\gamma_{0}^{2} a_{0}^{2} / 4$, $\psi\left(r_{s}\right)=0$ has three unequal real roots: $r_{s 3}<r_{s 2}<r_{s 1}=1$.

(c2.3) if $p=1-\gamma_{0}^{2}\left(N_{c}-a_{0}^{2} / 2\right)$, then $\psi\left(r_{s}\right)=0$ has three real roots: twofold root $r_{s 1}=r_{s 2}=1$ and $r_{s 3}<1$.

(c3) if $N_{c}<N_{c}^{*}$, if $N_{c}<N_{c}^{*}$, the following results aregiven:

(c3.1) if $p=1-\Lambda^{2} a_{0}^{2} \sqrt{N_{c}}-\Lambda a_{0}^{2} / 4, V\left(r_{s}\right)=0$ has three real roots: $r_{s 1}=1$ and $r_{s 2}=r_{s 3}=\sqrt{a_{0} / 2 \sqrt{N_{c}}}>1$. if $1-\Lambda_{0}^{2}\left(N_{c}-a_{0}^{2} / 2\right)<p<1-\Lambda^{2} a_{0}^{2} \sqrt{N_{c}}-\Lambda a_{0}^{2} / 4$, $V\left(r_{s}\right)=0$ has three unequal rael roots: $r_{s 1}=1<r_{s 3}<r_{s 2}$.

(c3.3) if $p=1-\gamma_{0}^{2}\left(N_{c}-a_{0}^{2} / 2\right), V\left(r_{s}\right)=0$ has three rael roots: twofold root $r_{s 1}=r_{s 3}=1$ and $r_{s 2}>1$.

\section{ACKNOWLEDGMENT}

The authors thank office of vice chancellor for research of Islamic Azad University, Bushehr Branch

\section{REFERENCES}

[1] V. I. Berezhiani and S. M Mhajan, Phys. Rev. Lett., vol. 73, pp. 1110-1113, 1994 
[2] T. Tatsuno, V. I. Berezhiani, and S. M Mahajan, Phys. Rev. E, vol. 63, pp. 046403-1-046403-7, 2011.

[3] D. Farina and S. V. Bulanov, Phys. Rev. Lett, vol. 86, pp. 5289- 5292, 2001.

[4] M. Lontano, S. Bulanov, and I Koga, Phys. Plasmas, vol. 8, pp. 5113-5120, 2001.

[5] D. Farina and S. V. Bulanov, Plasma Phys. Rep, vol. 27, pp. 680-692, 2001.

[6] M. Lontano, M. Passoni, and S. V. Bulanov, Phys Plasmas, vol. 10,pp 639-649, 2002.

[7] B. Eliasson and P. K. Shukla, Phys. Lett A, vol. 354, pp. 453-456, 2006.

[8] E. Heidari, M. Aslaninejad, and H. Eshraghi, Plasma Phys. Control. Fusion, vol. 53, pp. 075010-1-075010-14, 2010.

[9] P. K. Shukla and B. Eliasson, Phys. Rev. Lett, vol. 94, pp. 065002-1-065002-4, 2005.
[10] B. Eliasson and P. K. Shukla, JETP Lett., vol. 83, pp. 447-452, 2006. [11] Zhang et al., Phys. Plasmas, vol. 18, pp. 033104-1-033104-5, 2011.

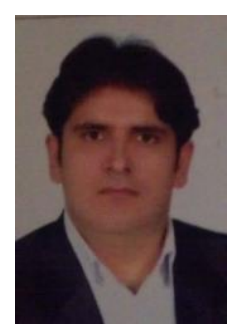

Ebrahim Heidari was born in Bushehr, Iran, at 1977. He earned B.A. in physics at Faculty of Physics, Shiraz University, Shiraz, Iran, by 2000. His M.S. in Atomic physics was finished at 2003 in the same university and his $\mathrm{Ph} . \mathrm{D}$. in plasma physics was earned at 2010 at Islamic Azad University, Science and Research Branch, Tehran, Iran. His major field of study is LaserPlasma interaction. He is ASSISTANT PROFESSOR of department of sciences, Islamic Azad University, Bushehr Branch, Bushehr, Iran. 